\title{
Lattice QCD with 12 Degenerate Quark Flavors
}

\author{
Xiao-Yong Jin* \\ Department of Physics, Columbia University, New York, NY 10027, USA \\ E-mail: xj2106@columbia.edu
}

Robert D. Mawhinney

Department of Physics, Columbia University, New York, NY 10027, USA

E-mail: rdmephysics.columbia.edu

\begin{abstract}
We report on new data from additional zero temperature simulations of QCD with 12 flavors. This is a continuation of previous studies using the DBW2 gauge action and naive staggered fermions. With the use of the force gradient integrator and a multiple-quark-mass preconditioned $\mathrm{HMC}$, we have done simulations with input quark masses from $m_{q}=0.003$ to $m_{q}=0.008$. We have observed a metastable, first order, bulk transition that occurs at small input quark masses. As the quark mass increases, this first order bulk transition ends at a second order critical point, and, for still heavier quark masses, becomes the cross-over we have previously reported. We present measurements of hadron masses, decay constants and other low energy observables in the small quark mass region on the weak coupling side of the bulk transition. Our results show that the behavior of the system is still consistent with spontaneously broken chiral symmetry. We also discuss a preliminary investigation into the behavior of the bulk transition itself. We have found that, as the system approaches the second order critical end point, the scalar singlet meson becomes lighter. Thus it appears that the critical endpoint corresponds to a continuum limit theory only involving scalars and, following known triviality arguments, this is likely a free field theory. The presence of this critical endpoint could influence scaling of lattice observables in the conventional continuum limit.
\end{abstract}

The XXIX International Symposium on Lattice Field Theory - Lattice 2011

July 10-16, 2011

Squaw Valley, Lake Tahoe, California

\footnotetext{
* Speaker.
} 


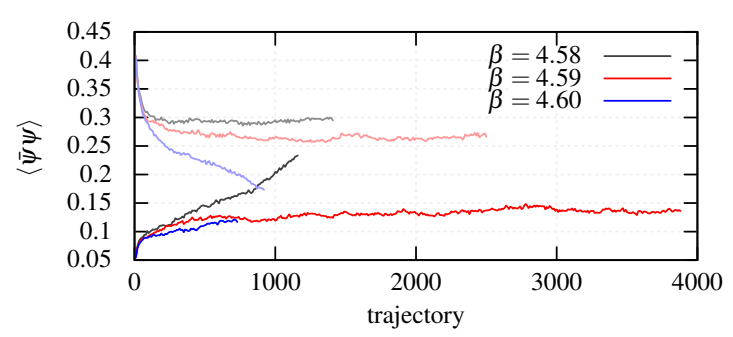

(a) 8 flavors, $m_{q}=0.015$

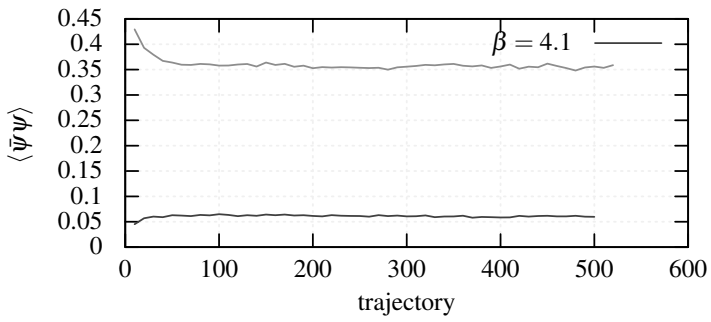

(b) 12 flavors, $m_{q}=0.01$

Figure 1: The evolution of $\langle\bar{\psi} \psi\rangle$ with 8 quark flavors (left) and 12 quark flavors (right), containing ordered (bottom curves) and disordered (top curves) starts, both with a lattice size of $16^{3} \times 32$.

\section{Introduction}

The zero temperature phase of QCD with many degenerate massless quark flavors has been a heated topic for a number of years. It is important to establish if an infrared fixed point is dynamically generated by a non-abelian gauge theory. It enables all the theoretical possibilities with a conformal symmetry (if the fixed point exists) or a walking coupling constant (if the number of flavors is less than required for a fixed point), which leads to some exciting theories as candidates for beyond the standard model physics.

Lattice methods are indispensable for determining the critical number of quark flavors required for the fixed point, as it is a non-perturbative phenomenon. Various groups are working on the subject. As the situation is new and unlike QCD in nature, we need to be careful in our interpretations of the numerical results from lattice calculations.

We have been using the DBW2 gauge and the naive staggered fermion action to simulate the SU(3) gauge theory with 8 and 12 flavors. In this report, we will discuss our investigation into a first order bulk transition separating strong and weak couplings, for small quark masses, and the second order critical point, where one expects the bulk transition to end, as the quark mass is increased. We argue that such a critical point (a lattice artifact) can alter the scaling behavior of the hadronic observables, if simulations are done in regions of the parameter space where the critical point has influence, and may have an impact on the running of the coupling constant directly measured on the lattice.

\section{First Order Bulk Transition}

A bulk transition has been found and studied in an earlier paper[1], for lattice QCD with 8 quark flavors, using the Wilson gauge action and naive staggered fermions, with the inexact $\mathrm{R}$ algorithm. The bulk transition was interpreted as a lattice artifact. It is independent of the lattice volume and temperature, and it is not associated with a deconfinement transition or chiral symmetry restoration. Recently, using the exact RHMC algorithm, we have rediscovered the bulk transition with the same gauge and fermion action as in the earlier study. We found it at $\beta=4.59$ and $m_{q}=0.015$ with a lattice size of $16^{3} \times 32$, shown in figure 1 a. Such a bulk transition, however, did not occur within the parameter space we have surveyed in our recent studies $[2,3,4]$ with the DBW2 gauge and naive staggered fermion action.

One might expect the lattice artifact bulk transition to become stronger with 12 quark flavors. The increase in the number of flavors makes the renormalized coupling run more slowly, and a higher lattice cutoff is required to approximate the continuum. In other words, at a comparable 


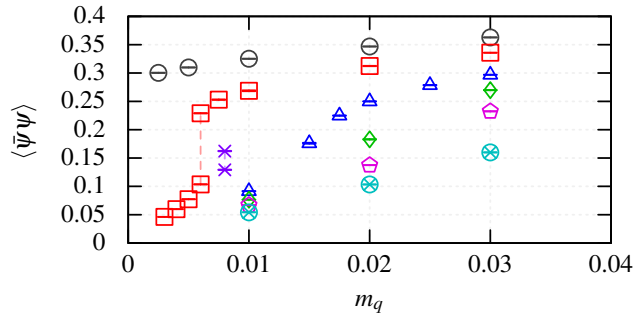

(a) $\langle\bar{\psi} \psi\rangle$

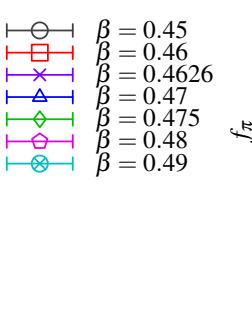

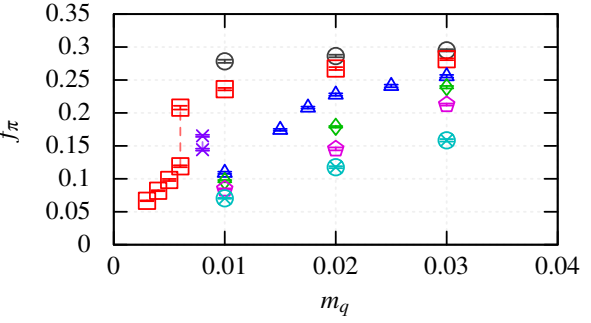

(b) $f_{\pi}$

Figure 2: $\langle\bar{\psi} \psi\rangle$ (left) and $f_{\pi}$ (right) with 12 flavors. Dashed lines indicates the meta-stable signal from the first order bulk transition, seen for both $\beta=0.46$ and 0.4626 .

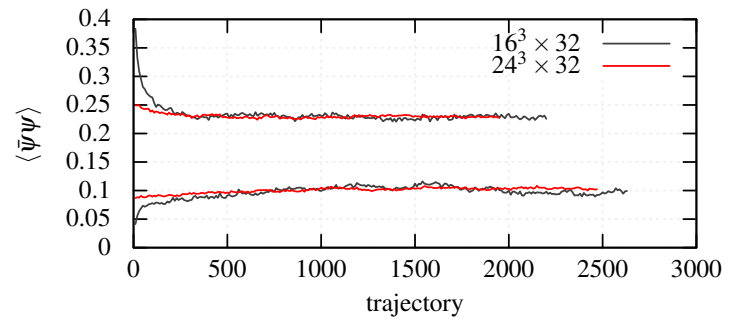

(a) $\beta=0.46, m_{q}=0.006$

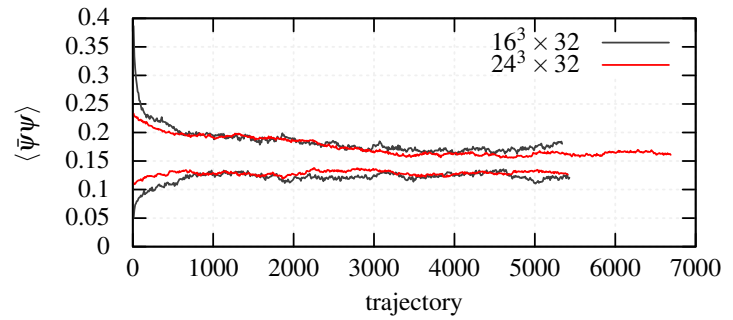

(b) $\beta=0.4626, m_{q}=0.008$

Figure 3: The evolution of $\langle\bar{\psi} \psi\rangle$ with DBW2 gauge and naive staggered fermion action. Two combinations of $\beta$ and $m_{q}$ show meta-stable evolutions with separate curves for ordered-starts (bottom curves) and disordered-starts (top curves). The metastability is present for two different volumes.

lattice spacing, the lattice gauge field becomes rougher with increasing number of flavors. The lattice consequently becomes a poorer approximation of the continuum. Observation of a bulk transition is mentioned in a study of a lattice Schrodinger functional calculation [5]. For 12 flavor QCD, with the same Wilson gauge and naive staggered fermion action, we have located the position of a first order bulk transition at $\beta=4.1$ and $m_{q}=0.01$ with a lattice size of $16^{3} \times 32$ and the exact HMC algorithm, as shown in figure $1 \mathrm{~b}$.

In our recent study of QCD with 8 and 12 flavors [3] with the DBW2 gauge and the naive staggered fermion action, it is intriguing to see that there is no meta-stability signal, but only a quark mass dependent, quick cross-over region. Nevertheless, the strong dependence on the quark mass suggests that a first order bulk transition may occur with smaller quark masses than we had been able to simulate with. Simulations with smaller quark masses became viable with algorithmic improvements. Using the force gradient integrator, which is accurate up to the $4^{\text {th }}$ order, combined with multiple Hasenbusch mass preconditioning, we were able to use the exact HMC algorithm to explore the smaller quark mass region.

A first order bulk transition was indeed found with 12 quark flavors at smaller quark masses, using the DBW2 gauge and naive staggered fermion action. The location of the bulk transition is shown in figure 2. It shows the first order, meta-stable points (those have two points at the same $\beta$ and $m_{q}$ ), along with other values ${ }^{1}$ from our earlier simulations. In the evolution of $\langle\bar{\psi} \psi\rangle$, shown in figure 3 , we have found meta-stable signals at two different couplings and quark mass

\footnotetext{
${ }^{1}$ In this paper, $f_{\pi}$ is normalized to be about $93 \mathrm{MeV}$ for QCD in physical units. Values of $f_{\pi}$ in our previous reports $[3,4]$ are off by a factor of 2 , because of a misunderstanding of the convention of the CPS.
} 
combinations. The meta-stable evolution lasted for a few thousand trajectories. This first order bulk transition has no visible volume dependence, as seen from comparing two different volumes, $16^{3} \times 32$ and $24^{3} \times 32$. Therefore, it is unlikely to be related to the thermal transition. In addition, as discussed previously [3], there is no visible change in the values of Polyakov loops, and there are clearly non-zero string tensions found in the inter-quark potentials at both sides of the transition. We can conclude that this bulk transition is not a deconfinement transition. On the other hand, the chiral symmetry is spontaneously broken on both sides of the bulk transition, as argued in our previous report [3]. The signal from all the hadronic observables is clear and well justified at the strong coupling side of the transition. On the weak coupling side, the linearity of $m_{\pi}^{2}$ versus $m_{q}$ suggests the existence of a dimensionful quantity $B$, as in leading order chiral perturbation theory where $m_{\pi}^{2}=2 B m_{q}$. Consequently, with a non-zero $f_{\pi}$ in the chiral limit, the GMOR relation, $f_{\pi}^{2} m_{\pi}^{2}=2 m\langle\bar{\psi} \psi\rangle$, predicts a small but non-zero chiral condensate in the chiral limit, which is consistent with out data. This argues that the weak coupling side of the bulk transition also has the chiral symmetry spontaneously broken. More of the spectrum results will be shown in the next section. In conclusion, the bulk transition does not restore chiral symmetry from the data we have collected.

Our simulations suggest the following phase structure. There is a first order bulk transition line in the $\beta$ versus $m_{q}$ plane, at smaller masses, $m_{q}<m_{q}^{C}$. At larger masses, $m_{q}>m_{q}^{C}$, the bulk transition becomes a mass dependent rapid cross-over. In addition to the usual Gaussian fixed point at $\beta=\infty$ and $m_{q}=0$, where the lattice gauge field approaches the continuum limit asymptotically, the results from our study of the system with 12 quark flavors suggest that there is an additional critical point at $\beta=\beta^{C}$ and $m_{q}=m_{q}^{C}$. It is the end point of the first order bulk transition, a second order phase transition point, where certain correlation lengths diverge. It also represents a continuum theory.

The position of the critical point depends on the lattice action. In our case, the change from the unimproved Wilson gauge to the DBW2 gauge action moves the critical point to smaller quark masses in lattice units.

With 8 flavors, it is also possible that the bulk transition does not go away completely, after we have switched to DBW2 from unimproved Wilson gauge. The bulk transition and the second order critical point may persist at a smaller quark masses away from the quark mass region we have analyzed. This possibility, however, does not invalidate our conclusions about the phase of the QCD system with 8 flavors. The spontaneous breaking of chiral symmetry and the asymptotic freedom holds at both side of the bulk transition.

Nevertheless, it is plausible that this additional critical point (different from $\beta=\infty$ ) is capable of affecting the scaling of the hadronic variables and also changing the running of the coupling constant directly measured on the lattice. More careful and thorough studies are needed to investigate the effect on the running of the coupling constant. Yet, continuing our recent studies, we are able to identify, detailed in the next section, the effect of this bulk transition critical point in the meson spectrum.

\section{The Meson Spectrum and the Scalar Singlet Meson}

Results for some meson masses are shown in table 1 for $\beta=0.46$ and $\beta=0.4626$, respectively, at $m_{q} \leq m_{q}^{C}$. Wall2Z sources are used for the propagators, except for $f_{\pi}$, which we measured from 


\begin{tabular}{llllllll}
$\beta$ & $m_{q}$ & Size & $f_{\pi}$ & $m_{\pi}$ & $m_{\sigma^{c}}$ & $m_{\rho}$ & $m_{a_{1}}$ \\
\hline \hline \multirow{6}{*}{0.46} & \multirow{2}{*}{0.003} & $24^{3} \times 32$ & $0.06782(74)$ & $0.19462(42)$ & $0.3429(37)$ & $0.4458(41)$ & $0.566(18)$ \\
& & $32^{3} \times 32$ & $0.06661(54)$ & $0.19370(33)$ & $0.3418(25)$ & $0.4338(34)$ & $0.585(11)$ \\
\cline { 2 - 7 } & 0.004 & $24^{3} \times 32$ & $0.08192(71)$ & $0.21633(53)$ & $0.3945(43)$ & $0.5016(62)$ & $0.638(27)$ \\
\cline { 2 - 8 } & \multirow{2}{*}{0.005} & $16^{3} \times 32$ & $0.0947(12)$ & $0.23519(91)$ & $0.436(15)$ & $0.589(10)$ & $0.96(10)$ \\
& & $24^{3} \times 32$ & $0.0983(16)$ & $0.23278(53)$ & $0.4811(99)$ & $0.6193(97)$ & $0.847(83)$ \\
\cline { 2 - 8 } & $0.006^{O}$ & $24^{3} \times 32$ & $0.1191(14)$ & $0.24368(28)$ & $0.565(16)$ & $0.756(12)$ & $0.995[94]$ \\
& $0.006^{D}$ & $24^{3} \times 32$ & $0.2080(28)$ & $0.21068(14)$ & $0.882(97)$ & $1.151[13]$ & $1.61[51]$ \\
\hline \multirow{2}{*}{0.4626} & $0.008^{O}$ & $24^{3} \times 32$ & $0.1442(15)$ & $0.26973(18)$ & $0.6667[86]$ & $0.8444[49]$ & $1.099[37]$ \\
& $0.008^{D}$ & $24^{3} \times 32$ & $0.1652(16)$ & $0.25926(16)$ & $0.773(16)$ & $0.986[16]$ & $1.226[52]$
\end{tabular}

Table 1: Some of the meson masses measured in the small quark mass region.

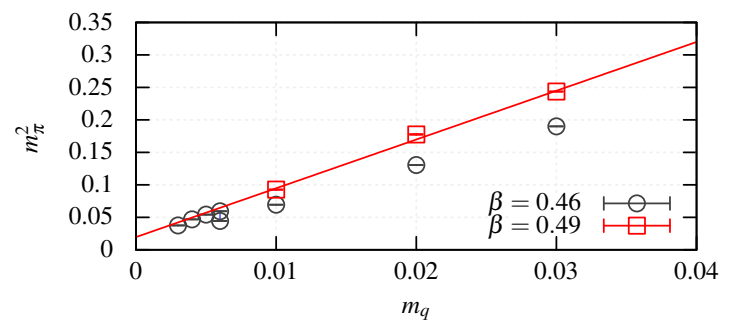

(a) $m_{\pi}^{2}$ versus $m_{q}$

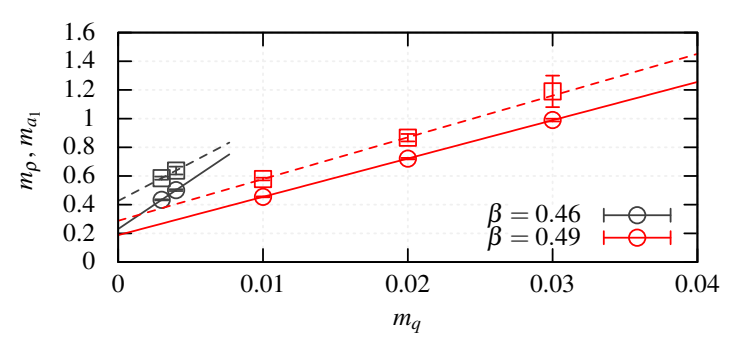

(b) $m_{\rho}$ and $m_{a_{1}}$ versus $m_{q}$

Figure 4: Masses of the $\pi, \rho$ and $a_{1}$ mesons versus input quark mass, $m_{q}$, at $\beta=0.46$ and $\beta=0.49$.

point to point propagators. In the table, the lable ${ }^{O}$ or ${ }^{D}$ on the quark mass indicates an ordered (weak coupling side) or disordered (strong coupling side) start, respectively. Most of the errors are statistical, except those enclosed by angular brackets have been increased to include a systematic variation caused by varying the fitting range.

Figure $4 \mathrm{a}$ shows that, on both sides of the bulk transition, there is the profound linearity of $m_{\pi}^{2}=2 B m_{q}$. This suggests that the chiral symmetry is spontaneously broken on both sides of the bulk transition. It is confirmed in the mass splitting between $\rho$ and $a_{1}$ mesons, as depicted in figure $4 b$.

There is still one thing left unexplained. It is well known that the correlation length diverges as a system approaches a second order critical point, which means some mass in the system with 12 flavors should vanish at the critical point. We can see from table 1 that, while the system goes from strong to weak coupling through the bulk transition, in lattice units, the masses of $\sigma, \rho$ and $a_{1}$ mesons drop, and the pion mass increases. This means that, going from strong to weak coupling, the lattice constant or the scale of the system decreases. However, none of the masses here appears to be vanishing toward the critical point, from $\beta=0.46, m_{q}=0.006$, to $\beta=0.4626, m_{q}=0.008$.

With further study, we have found that it is the scalar singlet meson whose mass appears to be vanishing near the critical point. Remember that the $\sigma$ meson masses shown in table 1 are only from the connected propagators. To measure the disconnected scalar propagators, we employed random Z2 volume sources. We have found marginal advantages with the Z2 against the $\mathrm{U} 1$ random source. We used 40 random sources for each gauge configurations to estimate the quark loops with $G(\tau)=\left\langle m \phi^{\dagger} \phi\right\rangle$, as proposed by the authors of the paper [6], where $M \phi=\eta$ with $\eta$ the random source and $M$ the fermion matrix. The singlet propagators, then, can be assembled as

$$
C(t)=\left\langle\frac{N_{f}}{4}\langle G(\tau) G(\tau+t)\rangle_{\tau}-C^{c}(t)\right\rangle-\frac{N_{f}}{4}\left\langle\langle G(\tau)\rangle_{\tau}\right\rangle^{2} \longrightarrow A\left(e^{-m t}+e^{-m\left(n_{\tau}-t\right)}\right)
$$




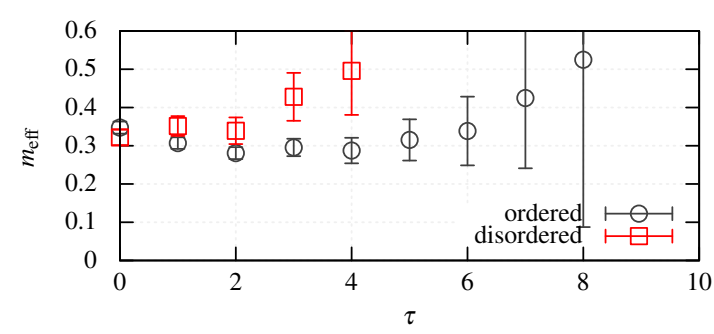

(a) $\beta=0.46, m_{q}=0.006$

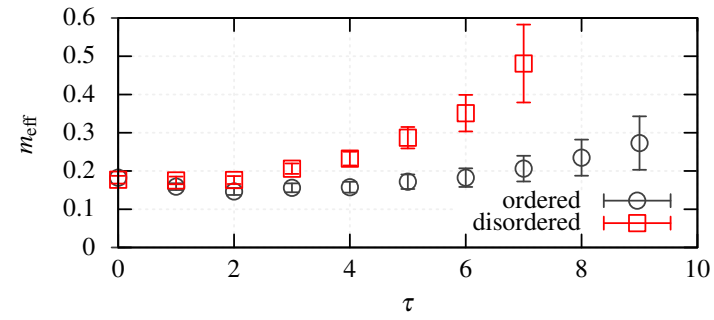

(b) $\beta=0.4626, m_{q}=0.008$

Figure 5: Effective masses of the scalar singlet meson. Gauge configurations with ordered and disordered starts respectively correspond to weak and strong coupling sides of the transition.

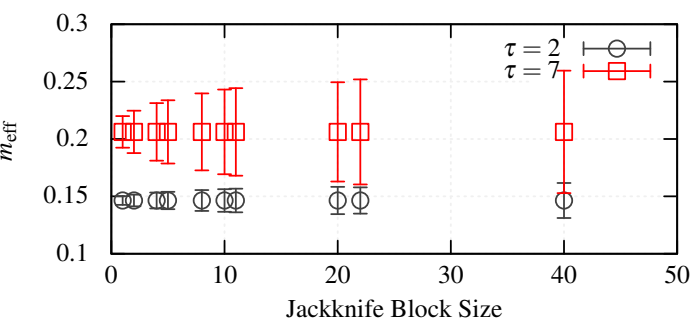

(a) Ordered-start, 440 configurations in total

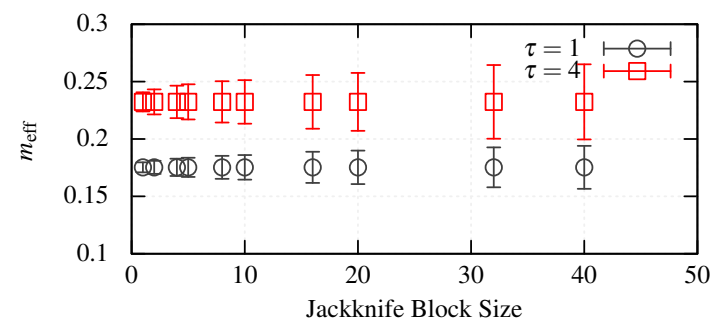

(b) Disordered-start, 320 configurations in total

Figure 6: Error of the effective masses of the scalar singlet meson versus the jackknife block size at $\beta=$ 0.4626 and $m_{q}=0.008$.

where $C^{c}(t)$ is the connected point to point propagator, $\langle\cdots\rangle_{\tau}$ is average over time slices, and $\langle\cdots\rangle$ is ensemble average. In the equation, a factor of $N_{f}$ is removed from the usual definition, and the factor of 4 comes from the staggered Dirac operator. We used a single mass state to fit the propagator to obtain the singlet mass, as the opposite parity state is numerically invisible to us.

Figure 5 shows the effective masses of the scalar singlet meson at the bulk transition. The value of $m_{\text {eff }}$ at $\tau$ is obtained from $C(\tau)$ and $C(\tau+1)$. It shows a bizarre behavior for all four propagators. When the temporal separation becomes larger, the effective mass starts increasing. However, it is understandable. The singlet propagator becomes noisier and more difficult to measure with increasing temporal separations. The expected behavior is demonstrated with the ordered-start configuration at $\beta=0.46$ and $m_{q}=0.006$, shown in figure 5a. We can see that although the expectation value increases with increasing $\tau$, the error increases at the same time.

The errors of $m_{\mathrm{eff}}$ are underestimated because of the long autocorrelation time. This is expected as the system approaches the second order critical point. As shown in figure 6, the error bar grows as one blocks the data in larger bins. It is important to keep in mind that our errors for the scalar single meson may be underestimated.

The mass of the scalar singlet meson was measured via fitting the propagators. The table at the left side of figure 7 shows the fitting range and the results. The statistical error quoted here were estimated using the jackknife method with a block size of 8 configurations. It is very exciting to see that the scalar singlet meson becomes really light at the point of the bulk transition at $\beta=0.4626$ and $m_{q}=0.008$, since it is closer to the second order critical point. If we apply the scaling of universality class as $m_{\sigma^{s}} \sim C\left(m_{q}-m_{q}^{C}\right)^{1 / 2}$, shown in figure 7 , we can estimate the critical quark mass at the end point to be at $m_{q}^{C}=0.00883$ (45) from ordered-start configurations, and $m_{q}^{C}=0.00869(29)$ from disordered-start configurations. 


\begin{tabular}{llcl}
$\beta$ & $m_{q}$ & $\tau$ fit range & $m_{\sigma^{s}}$ \\
\hline \hline \multirow{2}{*}{0.46} & $0.006^{O}$ & $2 \sim 7$ & $0.295(30)$ \\
& $0.006^{D}$ & $1 \sim 4$ & $0.361(34)$ \\
\hline \multirow{2}{*}{0.4626} & $0.008^{O}$ & $2 \sim 7$ & $0.160(14)$ \\
& $0.008^{D}$ & $1 \sim 4$ & $0.183(11)$
\end{tabular}

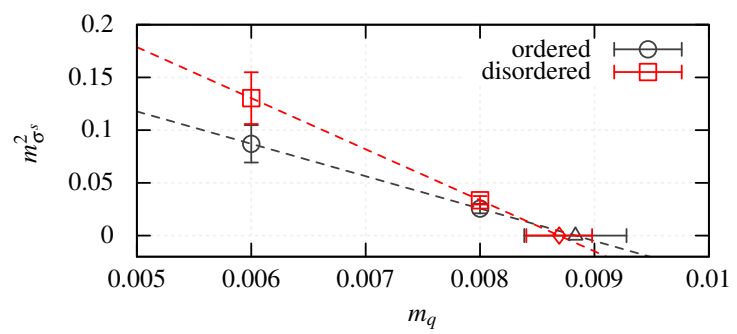

Figure 7: Masses of the scalar singlet meson at the first order bulk transition, and the extrapolations to the critical point.

\section{Conclusion}

We have found a first order bulk transition with 12 light quark flavors, using the DBW2 gauge and naive staggered action. All the hadronic observables are consistent with the system having spontaneously broken chiral symmetry and asymptotic freedom on both sides of the bulk transition.

The scalar singlet meson becomes lighter as the system approaches the second order critical point (the end point of the first order bulk transition), and is expected to have vanishing mass as other masses or hadronic scales remain finite at the critical point. This suggests that the continuum limit of the system at this critical point is a theory of scalars and hence one expects it to be a free theory, from the general arguments of the triviality of scalar field theories.

The scaling behavior of the hadronic observables is expected to be altered near this critical point, as opposed to the continuum limit governed by the Gaussian fixed point at $\beta=\infty$. Care must be exercised to do any scaling test. In particular, measuring the sigma mass while approaching the conventional continuum limit should indicate how much influence this critical endpoint has at a particular point in parameter space.

The running of the gauge coupling directly measured on the lattice is expected to be altered by this critical point, as it potentially deviates from the behavior in the weak coupling limit. More study is required to quantify this effect.

Our calculations were done on the QCDOC and NY Blue at BNL. X.-Y. Jin wants to express the sincere gratitude especially toward all the brilliant minds behind the QCDOC project. This research utilized resources at the New York Center for Computational Sciences at Stony Brook University/Brookhaven National Laboratory which is supported by the U.S. Department of Energy under Contract No. DE-FG02-92ER40699 and by the State of New York.

\section{References}

[1] F. R. Brown et al., Lattice QCD with eight light quark flavors, Phys. Rev. D46 (1992) 5655-5670 [arXiv:hep-lat/9206001].

[2] X.-Y. Jin and R. D. Mawhinney, Lattice QCD with Eight Degenerate Quark Flavors, POS (LATTICE 2008)059 [arXiv:0812.0413 [hep-lat]].

[3] X.-Y. Jin and R. D. Mawhinney, Lattice QCD with 8 and 12 degenerate quark flavors, PoS (LAT2009) 049 [arXiv:0910.3216 [hep-lat]].

[4] X.-Y. Jin and R. D. Mawhinney, Evidence for a First Order, Finite Temperature Phase Transition in 8 Flavor QCD, PoS (LATTICE 2010) 055 [arXiv:1011.1511 [hep-lat]].

[5] T. Appelquist, G. T. Fleming and E. T. Neil, Lattice Study of Conformal Behavior in SU(3) Yang-Mills Theories, Phys.Rev. D79 (2009) 076010 [arXiv: 0901.3766 [hep-ph] ].

[6] L. Venkataraman and G. Kilcup, The eta-prime meson with staggered fermions, Phys.Rev.D (1997) [arXiv:hep-lat/9711006 [hep-lat]]. 